This is the post peer-review accepted manuscript of:

M. Bin and L. Marconi, "The Chicken-Egg Dilemma and the Robustness Issue in Nonlinear Output Regulation with a Look Towards Adaptation and Universal Approximators," 2018 IEEE Conference on Decision and Control (CDC), Miami Beach, FL, 2018, pp. 5391-5396.

The published version is available online at:

https://doi.org/10.1109/CDC.2018.8619002

(C) 2018 IEEE. Personal use of this material is permitted. Permission from IEEE must be obtained for all other uses, in any current or future media, including reprinting/republishing this material for advertising or promotional purposes, creating new collective works, for resale or redistribution to servers or lists, or reuse of any copyrighted component of this work in other works. 


\title{
The Chicken-Egg Dilemma and the Robustness Issue in Nonlinear Output Regulation with a Look Towards Adaptation and Universal Approximators
}

\author{
Michelangelo Bin and Lorenzo Marconi
}

\begin{abstract}
In this paper we review the problem of output regulation for nonlinear systems. We discuss how the robustness properties and the simple structure of the linear regulator are linear artefacts which do not extend in more general nonlinear cases. We talk about the intertwining that is necessarily present between the internal model and the stabilising parts of a nonlinear regulator, in which the role of the exosystem mixes up with those of the residual plant's dynamics. We discuss a general guideline to deal with such structural challenges, by looking at adaptation and universal approximators as a promising way to provide systematic design procedures for approximate regulators in a general nonlinear setting.
\end{abstract}

\section{INTRODUCTION}

We consider a general class of continuous-time nonlinear systems of the form

$$
\begin{aligned}
\dot{x} & =f(w, x, u) \\
y & =h(w, x)
\end{aligned}
$$

with state $x \in \mathbb{R}^{n}$, control input $u \in \mathbb{R}^{m}$ and output $y \in \mathbb{R}^{q}$. The input $w \in \mathbb{R}^{n_{w}}$ is an exogenous signal that represents references to be tracked, disturbances to be rejected and unknown parameters. As customary in output regulation, we assume that $w$ is generated by a system of the form

$$
\dot{w}=s(w)
$$

called the exosystem. We associate to the plant (1) the regulation errors

$$
e=h_{e}(w, x) \in \mathbb{R}^{p}
$$

that are those variables on which the effect of the exogenous signal $w$ must be eliminated at the steady state. We suppose that $e$ belongs to the measured outputs ${ }^{1} y$, namely, that we can write $y=\left(e, y_{\mathrm{m}}\right)$, with $y_{\mathrm{m}} \in \mathbb{R}^{q-p}$ some additional outputs that have no steady-state requirements.

For the system (1), (2), (3) we consider the following approximate output regulation problem: given $\epsilon>0$ and sets $X \subset \mathbb{R}^{n}$ and $W \subset \mathbb{R}^{n_{w}}$ of initial conditions for (1), (2), find an output feedback regulator of the kind

$$
\begin{aligned}
& \dot{\zeta}=g(\zeta, y), \quad \zeta \in \mathbb{R}^{n_{\zeta}} \\
& u=\gamma(\zeta, y)
\end{aligned}
$$

Michelangelo Bin and Lorenzo Marconi are with CASY-DEI, University of Bologna, Bologna 40123, Italy (michelangelo.bin2@unibo.it and lorenzo.marconi@unibo.it). Research partially supported by the European Project AirBorne (G.A. 780960).

${ }^{1}$ This assumption is motivated by the fact that for linear systems the readability of $e$ from $y$ is necessary to obtain a robust design [1]. and a set $Z \subset \mathbb{R}^{n_{\zeta}}$, such that the closed-loop system (1), (2), (4) is locally uniformly eventually bounded from $W \times X \times Z$ and $\limsup _{t \rightarrow \infty}|e(t)| \leq \epsilon$. If $\epsilon=0$ we say that the regulation property is asymptotic, if $\epsilon>0$ can be made arbitrarily small, by opportunely tuning the regulator, we say that the regulation property is practical. For what concerns the sets $W, X$ and $Z$ : If $X=\mathbb{R}^{n}$ we say that the regulation property is global, if it can be taken arbitrarily large by opportunely tuning (4) we instead say that the regulation property is semi-global.

The case in which the plant (1) and the exosystem (2) are linear, with the exosystem is perfectly known, has been solved in a very elegant way in the seminal works [1], [2], [3], where the celebrated internal model principle has been formulated. The control design of [3] is summarized in section II. The linear regulator enjoys a very special property: if the regulator is tuned for a nominal plant (and for a nominal exosystem) to obtain asymptotic regulation, asymptotic regulation holds also for any perturbation of the plant's data $f$ and $h$ that does not destroy linearity and closed-loop stability. This robustness property is direct consequence of linearity and has not been extended so far to nonlinear systems (for a more detailed analysis about robustness the reader is referred to [4]). The main existing approaches for nonlinear systems (see e.g. [5], [6]) have been developed only for the quite limited contexts of square normal forms, and the control designs are strongly based on the knowledge of a model of the ideal steady state control action that is able to make the set in which the regulation error vanishes invariant ${ }^{3}$. This ideal steady-state control law strongly depends on the exosystem and the zero dynamics associated to the plant, and hence, any design based on its knowledge is in principle not robust to plant's or exosystem's perturbations.

In this paper we review the main reasons why the extension of the very special properties of the linear regulator to a nonlinear context is such a difficult task. In this way, we delineate in a very general framework what are the main structural difficulties and challenges that are behind the design of a robust nonlinear regulator. The idea that shines throughout the paper is that the robustness property and the simplicity of structure of the linear regulator are mere consequence of the strongest and dominant assumption

\footnotetext{
${ }^{2}$ Namely, for every bounded subset $U \subset W \times X \times Z$, there exists $M>0$ and $T>0$ such that, for every solution $(w, x, \zeta)$ originating in $U$ $|(w(t), x(t), \zeta(t))| \leq M$ for all $t \geq T$.

${ }^{3}$ This control action is usually called the friend and the design techniques that rely on it, or on its dynamic model, are said to be friend centric.
} 
of linearity, and that in a general context a neat separation among the different parts of the regulator is not possible and that the idea of robustness typically framed in the linear context might not be appropriate for nonlinear systems. In parallel to [4], which goes toward a more abstract idea of robustness, in this paper we look at a regulator structure that naturally embraces the different features that arise in nonlinear systems, with a look toward adaptive strategies and "universal approximators" seen as tools to deal with the nonlinear challenges.

\section{The LineAR REgulator}

In this section we consider the system (1), (2), (3) under the assumption that $p \leq m$ and the functions $f, s$ and $h$ are linear and, in some fixed basis of $\mathbb{R}^{n_{w}} \times \mathbb{R}^{n}$, we have

$$
\begin{aligned}
& \dot{w}=S w \\
& \dot{x}=A x+B u+P w \\
& y=\left(\begin{array}{c}
e \\
y_{\mathrm{m}}
\end{array}\right)=\left(\begin{array}{c}
C_{e} x+Q_{e} w \\
C_{\mathrm{m}} x+Q_{\mathrm{m}} w
\end{array}\right)=C x+Q w
\end{aligned}
$$

for some matrices $S, A, B, P, C$ and $Q$ of appropriate dimension, with $S$ that is assumed to be neutrally stable. Well known arguments (see e.g. [2]) show that necessary and sufficient conditions under which asymptotic regulation is possible is the existence of matrices $(\Pi, \Gamma)$ solving the following regulator equations:

$$
\begin{array}{ll}
\Pi S & =A \Pi+B \Gamma+P \\
0 & =C_{e} \Pi+Q_{e} .
\end{array}
$$

The functions $x^{\star}(t)=\Pi w(t)$ and $u^{\star}(t)=\Gamma w(t)$ represent respectively the ideal steady state trajectories for the state $x$ and the control input $u$ ensuring a zero regulation error. The linear regulator is obtained by letting in (4) $\zeta=(\eta, \xi)$ and $(g, \gamma)$ be such that

$$
\begin{aligned}
\dot{\eta} & =\Phi \eta+G e \\
\dot{\xi} & =H_{\xi} \xi+H_{\eta} \eta+H_{y} y \\
u & =K_{\xi} \xi+K_{\eta} \eta+K_{y} y
\end{aligned}
$$

where $n_{\eta}:=\operatorname{dim}(\eta)=p n_{w},(\Phi, G)$ is a controllable pair with the characteristic polynomial of $\Phi$ that equals those of $S$ and with $n_{\xi}:=\operatorname{dim}(\xi), H_{\xi}, H_{\eta}, H_{y}, K_{\xi}, K_{\eta}$ and $K_{y}$ that are chosen to stabilise the resulting closed-loop system. It can be proved that, as long as the origin of closed loop system is asymptotically stable with $w=0$, then the state $(x, \eta, \xi)$ reaches a steady state $\left(x^{\star}, \eta^{\star}, \xi^{\star}\right)$ with $x^{\star}(t)=\Pi w(t)$, $u^{\star}(t)=\Gamma w(t)$ and, hence, $e^{\star}(t):=C_{e} x^{\star}(t)+Q_{e} w(t)=0$.

A celebrated property of the linear regulator is that it is structurally robust. Namely, although uncertainties in the plant's data $A, B, P, C$ and $Q$ reflect into changes of the solution $(\Pi, \Gamma)$ to $(6)$ (and hence of the steady state value of $(x, u)$ ), as long as the regulator (7) maintains asymptotic stability of the closed-loop system the steady state is still characterised by the property that $e^{\star}(t)=0$. In other words, asymptotic regulation is achieved despite the presence of (sufficiently small) linear variations of the plant's data.

Nevertheless, the prefect knowledge of the exosystem dynamics (i.e. the knowledge of the characteristic polynomial of $S$ ) is instead necessary to obtain asymptotic regulation and also slight variations in $S$ from the nominal value destroy this property, even if the plant's data are perfectly known.

A further nice property is that the design of the different parts of the regulator (7) can be accomplished in a "straight" way. Namely, the internal model can be fixed just once the exosystem dynamics is known and thereafter the stabilising part $\xi$ can be chosen so that to ensure the closed-loop stability. In this respect, there is no intertwining in the design of the subsystems $\eta$ and $\xi$, with $\xi$ that depends on $\eta$ and with $\eta$ which is instead independent on $\xi$.

\section{NONLINEAR REGUlation}

In this section we consider again the system (1), (2), (3) when the functions $f, h$ and $s$ are arbitrary nonlinear functions. We first discuss why the robustness properties of the linear regulator can not be extended to nonlinear systems and then we talk about the intertwining that is present in the design of the internal model $\eta$ and the stabiliser $\xi$ subsystems when linearity does not hold.

\section{A. Why robustness is far to be possible?}

The key point that makes linear systems so special is that they do not distort linear input signals. When the linear closed-loop system (5), (7) is asymptotically stable and is excited by the input $w$ produced by a linear exosystem, at the steady state all the signals in the closed-loop will contain the same harmonics of $w(t)$. In other words, all the signals in the closed-loop systems can be generated by linear processes with the same dynamic properties of the exosystem. It is worth pointing out that the internal model should not necessarily be a model of the exosystem. Rather it should be a model of any process that can generate the ideal steady state control law $u^{\star}(t)$ that forces the regulation error to be zero. For linear systems $u^{\star}(t)$ can be generated by any process that has the same frequencies of the exosystem, and hence, the fact that $\Phi$ contains all the modes of $S$ turns out to be sufficient to ensure that $\eta$ has the internal model property. For nonlinear systems this fortunate property simply does not hold. This can be easily seen by considering, for example, a system of the form

$$
\begin{aligned}
& \dot{w}_{1}=w_{2} \\
& \dot{w}_{2}=-w_{1} \\
& \dot{x}_{1}=x_{2}+x_{3} \\
& \dot{x}_{2}=-x_{1}-\beta x_{2}+\epsilon x_{1}^{3}+w_{1}+x_{3} \\
& \dot{x}_{3}=u-x_{1} \\
& e=x_{3}
\end{aligned}
$$

with $\beta>0$ and $\epsilon \in \mathbb{R}$ small numbers. In this case, every regulator that ensures $e(t)=0$ for all $t \in \mathbb{R}_{+}$, also must ensure $\dot{e}(t)=0$ almost everywhere in $\mathbb{R}_{+}$. This means that $u(t)$ must compensate, at the steady state, the effect of $x_{1}(t)$ (restricted to the set in which $x_{3}=0$ ). Hence, a possible candidate of $u^{\star}(t)$ is the output of the following system

$$
\begin{array}{ll}
\dot{w}_{1}=w_{2}, & \dot{w}_{2}=-w_{1} \\
\dot{x}_{1}=x_{2}, & \dot{x}_{2}=-x_{1}-\beta x_{2}+\epsilon x_{1}^{3}+w_{1} \\
u^{\star}=x_{1} . &
\end{array}
$$


Therefore, any regulator solving the problem must embed a model of (8) inside the control loop to be able to generate $u^{\star}(t)$. Now, no matter how small $\beta$ and $|\epsilon|$ are, for sufficiently large initial conditions of $(w, x)$ the system (8) admits chaotic solutions (see [7]). This means that the information given by the exosystem (a simple harmonic in this case) is arbitrarily far to be sufficient to individuate a model for the desired $u^{\star}$, and the role of the exosystem in the internal model fades away and mixes up with the parts of the plant (in this case the dynamics of $x_{1}$ and $x_{2}$ ) that participate in the definition of the possible $u^{\star}(t)^{4}$. When the system is linear (take $\epsilon=0$ in (8)) and asymptotically stable, then the plant's residual dynamics ( $x_{1}$ and $x_{2}$ in this case) simply do not participate in the definition of $u^{\star}(t)$ unless changing its phase or amplitude. That means that no matter we chose $\beta>0$ or add linear terms to $(8)$, if $\epsilon=0$ and $\left(x_{1}, x_{2}\right)$ is stable, then $u^{\star}(t)$ can be always produced by the system

$$
\begin{aligned}
& \dot{\eta}_{1}=\eta_{2}, \quad \dot{\eta}_{2}=-\eta_{1} \\
& u^{\star}=\eta_{1}
\end{aligned}
$$

which is completely defined by the knowledge of the exosystem.

This example shows how in nonlinear systems the plant itself plays a strong active part in the definition of the law $u^{\star}(t)$, and hence, if a regulator is constructed to embed a copy of the process that generates $u^{\star}(t)$ then any arbitrarily slight perturbation in the plant, as well as in the exosystem, can in principle invalidate such an internal model, thus breaking the possibility of obtaining asymptotic regulation. This leads us to conclude the following important, even if probably trivial, fact: an internal model based regulator can in principle guarantee asymptotic regulation robustly only under perturbations of the plant or the exosystem that do not affect the process that generates $u^{\star}(t)$. Thus looking for a nonlinear regulator that is robust with respect to arbitrary, even though small, perturbations of the plant is not different than looking for a linear regulator that is robust also with respect to variations in the exosystem matrix $S$. Simply it is unlikely to exist (even in the linear case), and the very special robustness property of the linear regulator seems to be just a fortunate consequence of linearity that has no nonlinear analogous for general systems.

\section{B. The chicken-egg dilemma in nonlinear regulation}

Inspired by the linear regulator we consider candidate regulators of the kind (4) that are subdivided into an internal model unit and a stabiliser. In particular we let $\zeta=(\eta, \xi)$, with $\eta \in \mathbb{R}^{n_{\eta}}$ and $\xi \in \mathbb{R}^{n_{\xi}}$ that fulfils equations of the form

$$
\begin{aligned}
\dot{\eta} & =\Phi(\eta, e) \\
\dot{\xi} & =\varphi(\eta, \xi, y) \\
u & =\gamma(\eta, \xi, y) .
\end{aligned}
$$

Clearly, as in the linear case, the design of the stabiliser requires the knowledge of the internal model unit, since

\footnotetext{
${ }^{4}$ This reflects also in the fact that for general nonlinear systems, the solutions to the nonlinear equivalent of the regulator equations (6) cannot be expressed only in terms of $w(t)$, as instead is supposed in almost all the literature of output regulation so far (see [8] for a more extended treatise).
}

it is supposed to stabilise the cascade of the exosystem, the plant and the internal model unit. However, if a steady state $\left(w^{\star}, x^{\star}, \eta^{\star}, \xi^{\star}\right)$ must be reached such that $e^{\star}:=$ $h_{e}\left(w^{\star}, x^{\star}\right)=0$, necessarily we must have

$$
u^{\star}=\gamma\left(\eta^{\star}, \xi^{\star}, y^{\star}\right) \quad \text { a.e. }
$$

where $y^{\star}:=h\left(w^{\star}, x^{\star}\right)$. As a consequence, the ideal steady state $\eta^{\star}$, which for fixed $u^{\star}$ must give, together with $\left(\xi^{\star}, y^{\star}\right)$, a solution to (10), cannot be defined independently of $\xi^{\star}$. As $\eta^{\star}$ must be a solution to $\dot{\eta}=\Phi(\eta, 0)$, this means that the choice of $\Phi$ is necessarily dependent on $\gamma$ and $\varphi$, as the first appears explicitly in (10), and the second relates to $\xi^{\star}$. If the plant, the exosystem and the stabiliser are linear, this intertwining fades away, as no matter how $\eta^{\star}$ will depend on $\xi^{\star}$, it will always solve the equation $\dot{\eta}^{\star}=\Phi \eta^{\star}$, as long as $\Phi$ contains the exosystem frequencies. In the nonlinear case though this decoupling is not in general possible anymore, and the intertwining among the design of the stabiliser and the internal model unit manifests itself in what we call the chicken-egg dilemma: in order to fix the internal model unit we must first fix the stabiliser but, on the other hand, the stabiliser must be fixed to stabilise a system that includes the internal model and therefore relies on its knowledge.

Among the different design solutions to handle output regulation problems for purely nonlinear systems it is worth mentioning [5] and [6] (see also [9], [10], [11], [12], [13] and the references therein). Interesting enough, both the design dealt with the chicken egg dilemma in the context of minimum-phase square (i.e. $\operatorname{dim}(u)=\operatorname{dim}(e))$ normal forms, with $y=e$ and with a common solution structure which is referred to as pre-processing. The pre-processing design is composed of a stabiliser which processes the available measurement $e$ and produces a stabilising action $v=v(e)$. This stabilising action is fed into an internal model unit that thus has access to $e$ only throughout $v$. The output of the internal model unit then sums up with $v$ to produce the control input $u$. The pre-processing design is somewhat complementary to the structure that arises naturally in the linear regulator, which is called a post-processing structure. In the post-processing schemes the internal model unit is directly fed by the regulation errors and its state, as well as the output $y$, constitutes the inputs of the stabiliser which has to stabilise the resulting closed-loop system. The reason why both the designs of [14] and [6] have been cast as pre-processing designs should be probably sought in the fact that such structure allowed to avoid dealing with the chicken-egg dilemma for the class of systems considered. Nevertheless, the pre-processing structure reveals some structural properties that seem to be hard, at least conceptually, to be consistent with a control design able to deal with nonlinear systems more general than minimum-phase square normal forms having simple controllability properties. For instance it is not clear how to deal in a pre-processing scheme with non-square multivariable systems, where the dimension of $u$ is larger than those of $e$. In this case, indeed, the internal model unit would be driven by an input $v(e)$, which has the same information as $e$, but possibly a higher 
dimension. Moreover, there is not a clear road map to handle additional measured outputs $y_{\mathrm{m}}$ whenever they are needed for stabilisation but they do not need to vanish at the steady state. In fact, if $y_{\mathrm{m}}$ are needed to form the stabilising action $v$ (that in this case would be a functions also of $y_{\mathrm{m}}$, i.e. $v=v(y)$ ) the steady state value $y^{\star}$ of $y$ would be either filtered by the stabiliser or included in the definition of the internal model, thus making the design, in principle, more fragile. For further details on the properties of pre- and postprocessing schemes the reader is referred to [15], [16], [8]. For both the designs of [5] and [6], an equivalent postprocessing scheme has been found, respectively in [16] and [15]. Although the post-processing versions have a structure more likely suitable for further generalisations, the current designs have been applied only to the same class of problems that pre-processing schemes can deal with, with the further complication that the intertwining between the internal model and the stabiliser appears explicitly (i.e. $\Phi$ depends on $v$ ).

\section{Dealing with the Chicken-EgG Dilemma}

As the intertwining between the internal model unit and the stabiliser is a feature that seems to be hardly avoidable whenever general nonlinear systems are considered, a codesign strategy for the two systems appears to be the natural way to proceed. In this section we discuss, at a high level, the main steps that a possible iterative co-design strategy needs to follow in order to deal with the chicken egg dilemma in the design of nonlinear regulators. An interesting point that will be discussed in the next section is that adaptation turns out to be a natural way to deal with the structural problems induced by the intertwining between the regulator components and the robustness issues, and it candidates as a prominent way to solve the chicken-egg dilemma in a quite general sense. In this respect, we also talk about "universal approximators" as an appealing way to construct systematic procedures for practical regulators. The use of adaptation and universal approximators in the control loop is further motivated in view of the robustness issues discussed in Section III-A, which led us to think that approximate or practical regulation objectives seems to be more suitable tasks in nonlinear contexts, and that adaptation, in the system identification terms, means also giving a meaning to the residual bound on the regulation errors in terms of prediction error ${ }^{5}$.

\section{A. The class of internal models}

By following the linear systems philosophy, the first step would consist in choosing a class of possible internal model units. As a different system structure will be able, in principle, to deal with a different classes of problems, we stress that the class of internal model units must be chosen according to the a-priori knowledge of the plant and exosystem. By way of example in this paper we choose a system structure inspired by the post-processing version [16] of the high-gain design of [5]. We remark though that all the other designs, such as the Luenberger-like approach of [6], can be used

\footnotetext{
${ }^{5}$ For further details in this relationship between output regulation and system identification the reader is referred to [17], [8].
}

as well, with minor adjustments. With $d \in \mathbb{N}$ an arbitrary integer, let $\eta \in \mathbb{R}^{p d}$ be decomposed as $\eta=\left(\eta_{1}, \ldots, \eta_{d}\right)$, with $\eta_{i} \in \mathbb{R}^{p}$ fulfilling

$$
\begin{aligned}
& \dot{\eta}_{i}=\eta_{i+1}+G_{i} e \quad i=1, \ldots, d-1 \\
& \dot{\eta}_{d}=\psi(\eta)+G_{d} e,
\end{aligned}
$$

with $G_{i} \in \mathbb{R}^{p \times p}$ and $\psi: \mathbb{R}^{p d} \rightarrow \mathbb{R}^{p}$ to be fixed. The degrees of freedom in this design are: the dimension $d$, the matrices $G_{i}$ and the function $\psi$ and they will be fixed later by exploiting some additional knowledge on the stabiliser. It is worth noting that (11) extends the linear regulator as it can implement (up to a possible change of coordinates) the system (7) whenever $G:=\operatorname{col}\left(G_{1}, \ldots, G_{d}\right)$ and $\psi$ is linear.

\section{B. The class of stabilisers}

The stabiliser subsystem must be designed to stabilise the closed-loop system to the ideal steady state in which the regulation errors vanish. As a second step, a class of stabilisers must be fixed, and this task has to be done on the basis of the a-priori knowledge of the plant and exosystem and on the class of internal model units chosen at the previous stage. The information about the plant and exosystem comes into play as the functions $s, f$ and $h$ define an ideal steady state value of the control input that the regulator must produce. In particular we suppose that for each solution $w$ of interest to the exosystem there exists locally Lipschitz functions $x^{\star}: \mathbb{R}_{+} \rightarrow \mathbb{R}^{n}$ and $u^{\star}: \mathbb{R}_{+} \rightarrow$ $\mathbb{R}^{m}$ satisfying the following regulator equations:

$$
\begin{array}{lll}
\dot{x}^{\star} & =f\left(w, x^{\star}, u^{\star}\right) \\
0 & =h_{e}\left(w, x^{\star}\right) . & \text { a.e. }
\end{array}
$$

The class of stabilisers must be chosen so that, for any solution $\left(x^{\star}, u^{\star}\right)$ to $(12)$, the following consistency equations hold

$$
\begin{aligned}
& \dot{\xi}^{\star}=\varphi\left(\eta^{\star}, \xi^{\star}, y^{\star}\right) \\
& u^{\star}=\gamma\left(\eta^{\star}, \xi^{\star}, y^{\star}\right), \quad \text { a.e. }
\end{aligned}
$$

for some $\xi^{\star}: \mathbb{R}_{+} \rightarrow \mathbb{R}^{n_{\xi}}$ and $\eta^{\star}: \mathbb{R}_{+} \rightarrow \mathbb{R}^{n_{\eta}}$ and with $y^{\star}:=h\left(w, x^{\star}\right)$. The existence (for each $\left(x^{\star}, u^{\star}\right)$ solving (12)) of a solution $\left(\xi^{\star}, \eta^{\star}\right)$ to (13) is a steady-state left invertibility property that is necessary for the regulator to generate the friend $u^{\star}$. For what concerns $\eta^{\star}$, it represents the ideal steady state of the internal model unit. As we fixed a structure of the kind (11), we must add to (13) the following consistency constraint:

$$
\dot{\eta}_{i}^{\star}=\eta_{i+1}^{\star}, \quad i=1, \ldots, d-1 .
$$

Equations (13), (14) must hold together. If they do not hold, either the internal model class or the stabiliser class should be changed. The constraint (14), is obtained by (11) by imposing the condition $e=0$ and by using only the "fixed" features of (11) represented by the "chain-of-integrators" structure.

\section{Co-design of the internal model unit and the stabiliser}

This stage is where the chicken-egg dilemma comes into play. Once fixed the class of internal model units and the stabilisers, we should pick a choice of the actual degrees of freedom $d, G_{i}, \psi, \varphi$ and $\gamma$ inside the chosen classes 
guaranteeing that the resulting closed-loop system converges (even approximately) to the desired steady state $\left(x^{\star}, \eta^{\star}, \xi^{\star}\right)$. As the choice of the class of the internal model units guides the definition of a class of stabilisers, in view of the invertibility conditions (13)-(14), the choice of the class of stabilisers leads to the definition of a class $\mathcal{C}_{\eta^{\star}}$ of possible ideal steady states that the internal model (11) must provide. We shall include in $\mathcal{C}_{\eta^{\star}}$ also the variations of $\eta^{\star}$ induced by the expected perturbations in the plant or in the exosystem. In the linear case, if the stabiliser is linear and the exosystem is nominal, then the class $\mathcal{C}_{\eta^{\star}}$ is included in the set of solutions to a system with the same modes as the exosystem and this information leads to a single natural choice of $d$ and $\psi$ ( $G$ can be chose to enforce controllability) that is able to generate all the possible elements $\eta^{\star}$ in $\mathcal{C}_{\eta^{\star}}$. In other words, in the linear case with a linear class of stabilisers, $\mathcal{C}_{\eta^{\star}}$ is what suffices to univocally fix the internal model inside the class of linear systems. For general nonlinear systems we cannot expect this feature to hold anymore and a perfect design of $d, G_{i}$ and $\psi$ need not to exist. Motivated by an approximate or practical regulation framework, we can though exploit the information given by $\mathcal{C}_{\eta^{\star}}$ (which is the nonlinear equivalent of the information that comes from the knowledge of the exosystem in the linear case) to pick a value for $d, G_{i}$ and $\psi$ that is the best possible relatively to the available information.

Once the internal model is fixed, the stabiliser is chosen inside the predefined class so that to stabilise the closed-loop system in such a way that the state $(w, \eta, \xi)$ gets as close as possible to the ideal resulting steady state $\left(x^{\star}, \eta^{\star}, \xi^{\star}\right)$. In more formal terms, consider the change of coordinates

$$
(x, \eta, \xi) \mapsto(\tilde{x}, \tilde{\eta}, \tilde{\xi}):=(x, \eta, \xi)-\left(x^{\star}, \eta^{\star}, \xi^{\star}\right)
$$

and let

$$
\varepsilon^{\star}:=\dot{\eta}_{d}^{\star}-\psi\left(\eta^{\star}\right)
$$

For compactness, let $\mathbf{x}:=(x, \eta, \xi), \mathbf{x}^{\star}:=\left(x^{\star}, \eta^{\star}, \xi^{\star}\right)$ and $\mathbf{x}:=(\tilde{x}, \tilde{\eta}, \tilde{\xi})$. In the new coordinates, we have

$$
\begin{aligned}
& \dot{w}=s(w) \\
& \dot{\tilde{\mathbf{x}}}=\tilde{\mathbf{f}}\left(\tilde{\mathbf{x}}, \mathbf{x}^{\star}, \varepsilon^{\star}\right) \quad \text { a.e. }
\end{aligned}
$$

with $\tilde{\mathbf{f}}\left(\tilde{\mathbf{x}}, \mathbf{x}^{\star}, \varepsilon^{\star}\right):=\left(f\left(w, \tilde{x}+x^{\star}, \gamma\left(\tilde{\eta}+\eta^{\star}, \tilde{\xi}+\xi^{\star}, h(w, \tilde{x}+\right.\right.\right.$ $\left.\left.\left.x^{\star}\right)\right)\right)-f\left(w, x^{\star}, \gamma\left(\eta^{\star}, \xi^{\star}, h\left(w, x^{\star}\right)\right)\right), \tilde{\eta}_{2}+G_{1} h_{e}(w, \tilde{x}+$ $\left.x^{\star}\right), \ldots, \tilde{\eta}_{d}+G_{d-1} h_{e}(w, \tilde{x}+x), \psi\left(\tilde{\eta}+\eta^{\star}\right)-\psi\left(\eta^{\star}\right)+\varepsilon^{\star}, \varphi(\tilde{\eta}+$ $\left.\left.\eta^{\star}, \tilde{\xi}+\xi^{\star}, h\left(w, \tilde{x}+x^{\star}\right)\right)\right)$. We note that, for every value of $\mathbf{x}^{\star}, \tilde{\mathbf{f}}\left(0, \mathbf{x}^{\star}, 0\right)=0$, i.e. for every $\mathbf{x}^{\star}$, the set $\mathcal{A}:=\{(w, \tilde{\mathbf{x}}) \in$ $\left.\mathbb{R}^{n_{w}} \times \mathbb{R}^{n+n_{\eta}+n_{\xi}}: \tilde{\mathbf{x}}=0\right\}$ is invariant for (16) as long as $\varepsilon^{\star}=0$. This suggests a choice of the internal model unit in such a way to make the expected $\varepsilon^{\star}$ as small as possible, and the stabiliser so that the system (16) is practically inputto-state stable (see [18]) relatively to $\mathcal{A}$ and with respect to the input $\varepsilon^{\star}$. In particular we ask the existence of class $\mathcal{K} \mathcal{L}$ function $\beta$, a class $\mathcal{K}$ function $\rho$ and a constant $\nu$ such that, the solutions to (16) satisfy ${ }^{6}$

$$
|(w(t), \tilde{\mathbf{x}}(t))|_{\mathcal{A}} \leq \beta\left(|(w(0), \tilde{\mathbf{x}}(0))|_{\mathcal{A}}, t\right)+\rho\left(\left|\varepsilon^{\star}\right|_{t}\right)+\nu
$$

\footnotetext{
${ }^{6}$ We denoted by $|(w, \tilde{\mathbf{x}})|_{\mathcal{A}}:=\inf _{\left(w^{\prime}, \tilde{\mathbf{x}}^{\prime}\right)}\left|(w, \tilde{\mathbf{x}})-\left(w^{\prime}, \tilde{\mathbf{x}}^{\prime}\right)\right|$ the usual distance of $(w, \tilde{\mathbf{x}})$ to the set $\mathcal{A}$ and by $\left|\varepsilon^{\star}\right|_{t}:=\sup _{s \in(0, t)}\left|\varepsilon^{\star}(s)\right|$.
}

Condition (17) permits to conclude that the asymptotic distance of $\mathbf{x}$ to the ideal steady state $\mathbf{x}^{\star}$ in which the error vanishes $^{7}$ is asymptotically bounded by the quantities $\varepsilon^{\star}$ and $\nu$. As defined by $(15)$, the quantity $\varepsilon^{\star}$ represents the mismatch between the chosen internal model unit (11) and the actual process that generates $\eta^{\star}$. As a matter of fact, if $\eta^{\star}$ solves (11) (i.e. if the internal model unit has the internal model property) then $\varepsilon^{\star}=0$. The quantity $\nu$ is a term that represents the ability of the stabiliser in driving the closedloop system to the ideal steady state value $\mathrm{x}^{\star}$. Therefore, if the internal model unit and the stabiliser are perfectly chosen, we can obtain $(w, \tilde{\mathbf{x}}) \rightarrow \mathcal{A}$, which implies $e \rightarrow 0$. In view of the complexity of the system, the unmodelled dynamics in both the plant and the exosystem and the parametric or structural uncertainties, we cannot expect, outside the linear case, to be able to chose the internal model unit and the stabiliser so that $\varepsilon^{\star}=0$ and $\mu=0$. Nevertheless, approximate or even practical regulation problems fit into this framework and, as it will be delineated in the next section, naturally lead to adaptive solutions.

\section{AdAPtation And Universal Approximators}

Once the class of stabilisers is fixed as in Section IVB a class $\mathcal{C}_{\eta^{\star}}$ of possible candidate ideal steady states $\eta^{\star}$ is defined. Suppose to have fixed $d \in \mathbb{N}$, the $G_{i}$ 's, $\psi$ and the stabiliser and assume that there exists a known function $\sigma: \mathbb{R}^{d p} \rightarrow \mathbb{R}^{d p}$ such that, possibly after a transitory, we have

$$
\left|\sigma(\eta)-\eta^{\star}\right| \leq \mu
$$

for some "small" $\mu>0$. If (18) holds, we can use $\sigma(\eta)$ as an approximate measurement of $\eta^{\star}$ and we can look at (15), better rewritten as

$$
\dot{\eta}_{d}^{\star}=\psi\left(\eta^{\star}\right)+\varepsilon,
$$

as a prediction error model (see [19]). We can use the available information on $\eta^{\star}$ to adapt the function $\psi$ at runtime inside the class of internal model unit previously chosen. Under this system identification point of view, the knowledge of the class $\mathcal{C}_{\eta^{\star}}$ can be used to chose the order $d \in \mathbb{N}$ and the model set, that is, the set of possible models $\psi$ that may produce a good prediction model for the signal in $\mathcal{C}_{\eta^{\star}}$. In this way we can "move" the internal model unit inside the predefined class to match at best the information available on the actual $\eta^{\star}$. The stabiliser might be possibly changed consequently inside the correspondent class.

The condition (18) can be obtained, for instance, by choosing $G_{i}:=g^{i} a_{i} I_{p}$, with $g>0$ a control parameter and $\left(a_{i}\right)_{i}$ a set of coefficients of a Hurwitz polynomial (see [11], [8]). This high-gain design guarantees (18) with $\sigma(\eta)=\eta$ and $\mu$ that can be arbitrarily reduced as $g$ is increased. The high-gain construction, and the corresponding nice property that $\eta$ is asymptotically close to $\eta^{\star}$ despite the $\psi$ chosen, has been exploited in [17] and [8] to construct adaptive internal

\footnotetext{
${ }^{7}$ By continuity, (17) also implies $\lim \sup _{t \rightarrow \infty}|e(t)| \leq$ $\rho\left(\limsup _{t \rightarrow \infty}\left|\varepsilon^{\star}(t)\right|\right)+\nu$, so that we directly obtain a bound on the regulation error.
} 
model schemes, the first in a hybrid framework for singleinput-single-output minimum-phase normal forms, and the second in a more general multivariable context by following essentially the same guideline discussed in this paper. Further adaptation techniques can be thought of to be cast in a two time-scales framework (see [20]), in which the adaptation of $\psi$ happens much slower than the underlying closedloop dynamics. This permits to separate the two problems as customarily done in singular perturbations literature, by looking at (16) as the boundary layer system and to the adaptation algorithm as the reduced one. This idea has been exploited, in slightly different terms, in [21] in the context of linear systems, where the identification algorithms is discrete-time.

Adaptation itself does not solve the chicken-egg dilemma, that is instead moved to a higher level. As a matter of fact, in order to choose the order $d$ and the model set of $\psi$ (and thus the identification algorithm) we still need to rely on the class $\mathcal{C}_{\eta^{\star}}$ (which is defined also by the stabiliser class) of possible steady states, as different data sets lead in general to different identification problems. What adaptation gives is instead a more flexible way to deal with the actual stabiliser chosen in the pre-defined class, by allowing the chance to obtain asymptotic regulation also in presence of uncertainties in the plant or exosystem and, more important, by ensuring an approximate regulation objective with a bound on the regulation error that is directly related to the performance of the identification algorithm (see [8] for further details). It is thus natural to wonder if there is an identification model set that is able to produce a meaningful model for arbitrary functions $\eta^{\star}$. This identification model is referred to as an universal approximator, as it would be able, in principle, to approximate the right model for any element of any class $\mathcal{C}_{\eta^{\star}}$. Examples of universal approximators might be wavelets as well as neural networks. Even though asymptotic regulation would require in principle an infinite order $d$ (as it is the case of wavelets and neural networks), what is appealing of universal approximators is a universal practical regulation result of the kind: for each system of the form (1), (2) and each $\epsilon>0$ there exists an order $d \in \mathbb{N}$ such that approximate regulation holds with an error bound of $\epsilon$. This result could be possible in view on basic approximation theorems (see e.g. [22], [23]) and could be obtained by increasing the complexity of the identification model to match the desired error bound.

\section{CONCLusions}

In this paper we discussed the main challenges that are present in the design of nonlinear regulators, by underlining the fact that nonlinear regulators in general cannot have the same robustness properties and simplicity of structure of the celebrated linear regulator. We presented a guideline for the co-design of internal model units and stabilisers and we discuss how adaptation and universal approximators could be a promising way to deal in general cases with the chickenegg dilemma. Many research directions are open in this respect, from the co-design of internal models and stabilisers able to ensure (17), to the design of identification schemes that can be used with them. In this respect, the introduction of universal approximators in the output regulation framework is a promising research direction which could provide a solution to the chicken-egg dilemma and a systematic design method for practical regulation.

\section{REFERENCES}

[1] B. A. Francis and W. M. Wonham. The internal model principle of control theory. Automatica, 12:457-465, 1976.

[2] B. A. Francis. The linear multivariable regulator problem. SIAM Journal on Control and Optimization, 15(3):486-505, 1977.

[3] E. J. Davison. The robust control of a servomechanism problem for linear time-invariant multivariable systems. IEEE Trans. Autom. Control, 21:25-34, February 1976.

[4] D. Astolfi, M. Bin, L. Marconi, and L. Praly. About robustness of internal model-based control for linear and nonlinear systems. 2018. accepted to 2018 IEEE 58th Conference on Decision and Control (CDC).

[5] C. I. Byrnes and A. Isidori. Nonlinear internal models for output regulation. IEEE Trans. Autom. Control, 49:2244-2247, 2004.

[6] L. Marconi, L. Praly, and A. Isidori. Output stabilization via nonlinear Luenberger observers. SIAM Journal of Control and Optimization, 45:2277-2298, 2007.

[7] J. C. Sprott. Elegant chaos. Algebraically simple Chaotic Flows. World Scientific, 2010.

[8] M. Bin and L. Marconi. Adaptive post-processing internal models design for MIMO minimum-phase nonlinear systems, 2018. Under review.

[9] M. Maggiore and K. M. Passino. Output feedback tracking: A separation principle approach. IEEE Trans. Autom. Control, 50(1):111-117, 2005.

[10] L. Marconi and L. Praly. Uniform practical nonlinear output regulation. IEEE Trans. Autom. Control, 53:1184-1202, June 2008.

[11] A. Isidori, L. Marconi, and L. Praly. Robust design of nonlinear internal models without adaptation. Automatica, 48:2409-2419, 2012.

[12] A. Isidori. Lectures in Feedback Design for Multivariable Systems. Springer International Publishing, 2017.

[13] L. Wang, A. Isidori, H. Su, and L. Marconi. Nonlinear output regulation for invertible nonlinear MIMO systems. Int. J. of Robust and Nonlinear Control, 26:2401-2417, 2016.

[14] C. I. Byrnes and A. Isidori. Limit sets, zero dynamics and internal models in the problem of nonlinear output regulation. IEEE Trans. Autom. Control, 48:1712-1723, 2003.

[15] A. Isidori and L. Marconi. Shifting the internal model from control input to controlled output in nonlinear output regulation. In 2012 IEEE 51st Annual Conference on Decision and Control (CDC), pages 4900-4905, Maui, Hawaii, USA, December 2012.

[16] M. Bin and L. Marconi. About a post-processing design of regressionlike nonlinear internal models. In Proc. 20th IFAC World Congress, Toulouse, France, July 2017.

[17] F. Forte, L. Marconi, and A. R. Teel. Robust nonlinear regulation: Continuous-time internal models and hybrid identifiers. IEEE Trans. Autom. Control, 62(7):3136-3151, 2017.

[18] Z.-P. Jiang, A. R. Teel, and L. Praly. Small-gain theorem for ISS systems and applications. Math. Control Signals Systems, 7:95-120, 1994.

[19] L. Ljung. System Identification. Theory for the User. Prentice Hall, Upper Saddle River, N.J., 1999.

[20] A. R. Teel, L. Moreau, and D. Neşić. A unified framework for inputto-state stability in systems with two time scales. IEEE Trans. Autom. Control, 48(9): 1526-1544, 2003.

[21] M. Bin, L. Marconi, and A. R. Teel. Results on adaptive output regulation for linear systems by least-squares identifier. 2018. accepted to 2018 IEEE 58th Conference on Decision and Control (CDC).

[22] G. Cybenko. Approximation by superpositions of a sigmoidal function. Math. Control Signals Systems, 2:303-314, 1989.

[23] I. Daubechies. Ten Lectures on Wavelets. SIAM, 1992. 